\title{
Diagnostic Accuracy of Cardiac Computed Tomographic Angiography and Transesophageal Echocardiography in Evaluation of Patients with Prosthetic Paravalvular Leakage
}

Amr Youssef ( $\sim$ amryoussef@kasralainy.edu.eg )

Cairo University Kasr Alainy Faculty of Medicine

Hussien Heshmat Kassem

Cairo University Kasr Alainy Faculty of Medicine

Mohamed Ali Salem

Cairo University Kasr Alainy Faculty of Medicine

Hossam Ibrahim Kandil

Cairo University Kasr Alainy Faculty of Medicine

\section{Research Article}

Keywords: Prosthetic heart valve dysfunction, Prosthetic paravalvular leakage, ECG gated Computed Tomography, Transesophageal echocardiography

Posted Date: February 3rd, 2022

DOI: https://doi.org/10.21203/rs.3.rs-1206358/v1

License: (c) (1) This work is licensed under a Creative Commons Attribution 4.0 International License.

Read Full License 


\section{Abstract}

\section{Introduction and purpose:}

Computed Tomography (CT) scan has been well addressed to provide a diagnostic information for patients with prosthetic heart valve dysfunction. However, its role in the assessment of patients with prosthetic paravalvular leakage (PVL) has not been studied thoroughly. So, this study was conducted to assess the feasibility, reproducibility, and accuracy of CT for diagnosis of prosthetic PVL using surgical findings as the reference standard.

\section{Methods and results:}

This was a prospective cohort study that was conducted on 26 consecutive patients with suspected prosthetic PVL who underwent both transesophageal echocardiography (TEE) and 64-slice ECG-gated CT. The gold standard was the intraoperative findings. Surgery was performed in twenty-six patients. There was an excellent degree of agreement between CT and intraoperative findings for diagnosis, localization of prosthetic PVL. The perimeter of prosthetic PVL measured by CT was strongly correlated with echocardiographic severity of PVL by TEE (Spearman's Correlation Coefficient, $r=0.83, P$ value $=0.0014$ ).

\section{Conclusion}

This study demonstrates that cardiac CT showed comparable diagnostic accuracy to TEE and intraoperative findings for the detection, localization, and assessment of severity of prosthetic PVL. Moreover, CT was shown to be useful in detection of other findings related to prosthetic cardiac valves.

\section{Introduction}

Paravalvular leakage (PVL) has an estimated prevalence 2-10\% after aortic valve replacement and 7-17\% after mitral valve replacement (MVR) [1, 2]. Although most PVLs are asymptomatic, but an estimated 1$5 \%$ of patients with PVLs show serious adverse outcome including reoperation, cardiac and noncardiac deaths, and recurrent admissions with heart failure symptoms [3-5].

Both transthoracic echocardiography (TTE) and transesophageal echocardiography (TEE) are commonly used as the first-line imaging modalities for PVL detection. TEE can be of additional diagnostic value especially for Prosthetic PVL in the mitral position $[6,7]$.

However, imaging distortions from acoustic shadowing has been considered as a major limitation for echocardiography in prosthetic valve evaluation, another limitation worth mentioning is the semi-invasive nature of TEE. 
Cardiac computed tomography (CT) has received attention for its role in the evaluation of prosthetic valves dysfunction [8]. Although cardiac CT had a role diagnostic value in the diagnosis and guidance of successful transcatheter closure of mitral PVL, Diagnostic performance of cardiac CT for detection of prosthetic PVL has not been studied in detail [9-12].

The purposes of our study were to assess the feasibility, reproducibility, and diagnostic accuracy of CT compared to TEE for the diagnosis of prosthetic PVL using surgical findings as the reference standard.

\section{Methods}

From March 2017 to March 2019, we prospectively enrolled all patients with clinically suspected prosthetic paravalvular dysfunction. (e.g., suspected prosthetic heart valve obstruction, endocarditis or paravalvular leak referred to the echocardiography laboratory. A total of eighty patients with suspected prosthetic valve dysfunction were enrolled. Twenty-six patients with prosthetic significant paravalvular leakage were included in the study, whereas fifty-four patients were excluded as mentioned in Figure (1).

\section{Exclusion criteria}

1. Patients who refused to participate in the study and refused to sign consent.

2. The usual contraindications to multidetector $\mathrm{CT}$ angiography including pregnancy and contrast allergy.

3. Patients whom labs revealed renal impairment. (Renal impairment defined as Estimated GFR $<45$ $\mathrm{ml} / \mathrm{min} / 1.73 \mathrm{~m}^{2}$, or serum creatinine $>1.3 \mathrm{mg} / \mathrm{dl}$ )

4. Patients presented with hemodynamic instability or requiring emergency surgery.

5. Patients with prosthetic heart valves (PHVs) known to have beam hardening artefacts (containing cobalt-chrome alloy rings e.g., Björk-Shiley and pyrolytic carbon tilting-disk PHVs) that prevent diagnostic assessment.

\section{Study population characteristics}

All patients were subjected to the following work up on initial presentation.

Clinical history including age, gender, comorbidities, previous heart valve surgeries, time since last valve surgery, symptoms suggestive of congestive heart failure, symptoms suggestive of infective endocarditis (i.e. fever, sepsis, previous antibiotic treatment before clinical presentation).

Physical examination including blood pressure, heart rate, temperature, auscultations for prosthetic valve sounds (mitral, aortic or both), murmurs of regurgitant lesions across prosthetic or native valves. Laboratory work up included complete blood count, C reactive protein (CRP), serology for Brucella, Bartonella, Coxiella, Aspergillus, blood culture and sensitivity, kidney function tests including blood urea nitrogen and serum creatinine. 
All echocardiographic studies were conducted with a Philips iE33 ultrasound system and X7-2t transesophageal transducer (Philips Medical Systems, Andover, MA, USA). Standard views for combined TTE and TEE were acquired by experienced non-invasive cardiologists. Cardiac chambers quantification was done according to the American Society of Echocardiography for assessment of cardiac chambers including calculation of left ventricle end diastolic and end systolic volume and diameter, septal and posterior wall thickness, right sided chamber volume and dimensions, aortic and left atrium diameter [13]. Assessment of prosthetic cardiac valves was done according to the American Society of Echocardiography for assessment of prosthetic cardiac valves [14].

Transesophageal echocardiography was done for better assessment of prosthetic cardiac valves specifically looking for:

- Paravalvular leak: Location, number, size of defect, severity of regurgitant jets depending on criteria for assessment prosthetic cardiac valves in American Society of Echocardiography [14].

- Other abnormalities of prosthetic cardiac valves including [15].

a. Abscess: defined as irregularly shaped, inhomogeneous paravalvular masses within peri annular region, myocardium, or pericardium

b. Pseudo-aneurysm: defined as a space filled with contrast medium with a communication with the cardiac chambers or the aortic root.

c. Vegetation: defined as irregularly oscillating masses, adherent to and distinct from the endocardium.

d. Fistula: defined as a continuation between the chambers of the left and right heart.

e. Prosthesis dehiscence: defined as a rocking motion of a prosthetic valve with an excursion $>15^{\circ}$ in any 1 plane.

\section{CT Acquisition and Image reconstruction}

All the cardiac computed tomographic angiography studies were done with the Aquilion 64 machine, (Toshiba Medical Systems, Nasu, Japan) which provides a 64- sections for optimal imaging. Our 64section multidetector CT protocol was based on retrospectively ECG gated CT aortography protocols.

Raw data were reconstructed into 10 equally spaced datasets within the R-R interval of the cardiac cycle and were loaded simultaneously into the dedicated cardiac analysis software (vitreous vital image). The imaging planes were aligned parallel with and perpendicular to the valve leaflets as well as in plane with the valve in three perpendicular imaging planes.

For contrast material- enhanced imaging, we planned the acquisition from $2 \mathrm{~cm}$ above the carina (including the ascending aorta) to the bottom of the heart to achieve complete imaging of the heart. When desired, the scanning range was reduced in the craniocaudal direction to reduce radiation exposure. 
For anatomic assessment, the best systolic and diastolic reconstruction phases were selected. For dynamic evaluation, cine images in the plane perpendicular to the valve leaflets were recorded after appropriate alignment and windowing.

All CT analyses were performed by 2 experienced radiologists who were blinded to the results of TTE and TEE. CT image quality was assessed and those patients who had bad image quality (presence of severe artifacts and non-diagnostic for appropriate evaluation of the prosthetic valves) were excluded.

The proposed report included several items:

- PHV:

- Type: Mechanical or biological

- Position: Mitral or aortic and/or both.

- Anatomical PHV assessment.

- Paravalvular regurgitation presence and location. We also measured the para-prosthetic anatomical regurgitant orifice area to determine the accuracy and reproducibility of Cardiac CT in assessment of prosthetic paravalvular regurgitation. The size of the PVL was assessed by measuring the perimeter on long-axis views and area on the short-axis view. When CT image quality was suboptimal and not assessable for PVL evaluation, PVL was considered absent for the purpose of data analysis $[16,17]$.

- Other prosthetic valves abnormalities: Vegetations, abscess, Pseudoaneurysm, thrombus.

- Dynamic PHV assessment.

- Normal opening and closing angle: Frame-by-frame analysis of a single cardiac cycle were the basics for defining opening and closing valve angles by CT. For single-leaflet valves, a valve was defined as stuck when motion of one valve leaflet was absent. For bileaflet valves, opening and closing angles were measured between the two leaflets in the fully open and closed positions. Prosthetic valve obstruction was diagnosed when motion of a leaflet or leaflets was persistently restricted, with a calculated opening angle of more (for bileaflet valve) or less (for disk valves) than the values for a normal valve, as specified by the manufacturer [18].

\section{Surgical data}

Twenty-six patients with significant prosthetic PVL underwent cardiac surgery. For patients who underwent redo-surgery, the presence of a PVL orifice was confirmed by inspection on the surgical view. All the patients had intraoperative TEE for identification of prosthetic PVL and other abnormalities of the cardiac valves.

The surgical data that were reported by the surgeon during the open-heart surgery were considered the gold standard for comparing the diagnostic accuracy of transesophageal echocardiography and cardiac computed tomographic angiography.

Surgical data included all the following: 
1. Paravalvular regurgitation: presence, location, and severity.

2. PHV Function: Opening and closure of the prosthetic valve during cardiopulmonary bypass surgery.

3. Other abnormalities: Vegetations, abscess, Pseudoaneurysm, thrombus.

\section{Statistical analysis}

Data were coded and entered using the statistical package SPSS (Statistical Package for the Social Science; SPSS Inc, Chicago, IL, USA) version 22. Data were described using the mean, standard deviation in continuous variables and using frequency (count) and relative frequency (percentage) for categorical data. Categorical variables are presented in numbers (percentages).

The agreements on the presence and location of PVL between CT and surgical findings and between TEE and surgical findings were analyzed using Cohen's kappa statistics. The correlation between the size of prosthetic PVL on CT (area and perimeter) and the severity on TTE or TEE was analyzed using Spearman's correlation coefficient. Probability values $<0.05$ were considered statistically significant. The diagnostic performance of CT and echocardiography for diagnosing prosthetic PVL was assessed using surgical findings as the standard reference.

\section{Results}

\section{Patient population}

Eighty patients with a suspected PHV dysfunction were enrolled. Twenty-six patients were eventually included in the study as shown in Figure (1).

\section{Population characteristics}

Our study populations had mean age $41 \pm 12$ years, with almost three quarters of patients were males.

Heart failure symptoms (dyspnea at rest, orthopnea, and paroxysmal nocturnal dyspnea) were the most common presenting symptoms, followed by symptoms suggestive of infective endocarditis (fever, embolic manifestations).

Table (1) summarized the results of laboratory workup for enrolled patients.

\section{Prosthetic Paravalvular leakage}

\section{Paravalvular leakage presence.}

CT versus TEE for diagnosing presence of PVL. There was excellent agreement between CT and TEE for diagnosing the presence of paravalvular leakage (agreement k: 0.96).

In this study there was one patient who was diagnosed to have paravalvular leakage across aortic prosthesis by TEE which was not revealed by MCTA and was proved intraoperatively that the patient 
didn't have paravalvular leakage. Sensitivity, specificity of TEE and CT for detecting presence of PVL is $100 \%, 95 \%$ and $100 \%, 100 \%$ respectively.

CT and TEE versus surgery. There was excellent agreement between TEE and CT versus surgery (Gold standard) for diagnosing presence of paravalvular leakage with Agreement k: 0.95, 1.0 respectively as shown in Table (2).

CT had better diagnostic accuracy as compared to TEE in diagnosis of aortic prosthetic paravalvular leakage with sensitivity and specificity $100 \%, 100 \%$ for CT and $95 \%, 100 \%$ for TEE respectively. In our study, TEE incorrectly diagnosed aortic prosthetic PVL in one patient with large aortic root abscess, on the other hand, CT excluded the presence of PVL across the aortic prosthesis, (finding confirmed intraoperatively).

Tachycardia and/or acoustic shadowing of mechanical prosthesis may explain the limitation of TEE in assessment of the PVL presence in this patient.

\section{Paravalvular leakage site and location.}

The localization of PVL on the surgical field was done in all patients who undergone surgery for PVL. Figure (2) illustrated localization and severity assessment of aortic prosthetic PVL by both imaging modalities compared to intraoperative finding (Gold standard). CT accurately identified the location of PVL in 25 patients (100\%). TEE correctly identified the location of the PVL in 24 of 25 patients (96\%). The weighted kappa values for agreement of PVL location with the surgical field were 1 for CT and 0.94 for TEE as shown in Table (2).

Both CT and TEE correctly revealed the location of PVL in 24 patients, although in 1 patient with aortic prosthetic PVL, TEE incorrectly predicted the location due to the acoustic shadowing caused by the mitral prosthesis in this patient.

\section{Paravalvular leakage severity}

The perimeter of prosthetic PVL measured on CT was a mean of $0.63 \pm 0.15 \mathrm{~cm}$, while the vena contracta as a semiquantative measure for assessment of the prosthetic PVL measured by TEE was a mean of $0.62 \pm 0.2$.

Figure (3) illustrated quantitative methods used in both imaging modalities to assess severity of mitral prosthetic paravalvular leak in patient presented with heart failure symptoms. Table (3) showed a strong correlation between the perimeter of prosthetic PVL measured by CT and echocardiographic severity of PVL by TEE (Spearman's Correlation Coefficient, $r=0.83, P$ value $=0.0014$ ).

\section{Other abnormalities}

CT had lower sensitivity (75\%) for detection of vegetation as compared to TEE (88\%), both being compared to intraoperative findings. On the other hand, both modalities had $100 \%$ specificity for 
diagnosis presence of vegetation.

Both imaging modalities diagnosed aortic root abscess in seven patients with sensitivity and specificity of $100 \%$ for both.

Occluder malfunction of prosthetic valves were evaluated by both echocardiography (TTE and TEE) and cardiac computed tomographic angiography. The intraoperative assessment of occluder motion was considered the gold standard. The sensitivity and specificity of both modalities for diagnosing occluder malfunction were $100 \%$.

\section{Discussion}

Cardiac computed tomography (CT) has received attention for its role in the evaluation of prosthetic valves dysfunction [8]. Although cardiac CT had a role diagnostic value in the diagnosis and guidance of successful transcatheter closure of mitral PVL, Diagnostic performance of cardiac CT for detection of prosthetic PVL has not been studied in detail [9-11].

\section{Paravalvular leakage presence}

CT had better diagnostic accuracy as compared to TEE in diagnosis of aortic prosthetic paravalvular leakage with sensitivity and specificity were $100 \%, 100 \%$ for CT and were $95 \%, 100 \%$ for TEE respectively. In our study, TEE incorrectly diagnosed aortic prosthetic PVL in one patient with large aortic root abscess, on the other hand, CT excluded the presence of PVL across the aortic prosthesis (finding confirmed intraoperatively). This patient had undergone surgery for persistent infection due to a large aortic root abscess that was not responding to antibiotic therapy.

The diagnostic accuracy for PVL presence in our study was closely correlated to the findings of Hara et al. who reported perfect agreements between MDCT, Echocardiography, and surgical findings in aortic prosthetic regurgitation (APR) [16].

Sensitivity and specificity of both imaging modalities in diagnosis of PVL were closely correlated to that reported by Suh et al. who studied diagnostic performance of both CT and echocardiography compared with surgical findings as the standard reference for detection of mitral PVL with sensitivity and specificity were $96.9 \%$ and $97.8 \%$ respectively for CT, $96.2 \%$ and $95.8 \%$ respectively for TEE [17].

Our Study was different from the above-mentioned studies being prospective, on the other hand, Suh et al. was a retrospective one. In our study, we enrolled patients with either aortic and/or mitral prosthetic PVL, while Hara et al. and Suh et al. studied aortic and mitral prosthetic PVL respectively $[16,17]$.

\section{Paravalvular leakage location}

In most of the cases in our study, cardiac CT provided accurate information on PVL anatomy comparable to TEE. Also, CT may have additional value to TEE when the TEE images are not satisfactory for 
evaluation because of acoustic shadows. In one case in which TEE could not detect or correctly predict the location of PVL, CT correctly identified the PVL location. And this highlighted the importance of preprocedural imaging plays in providing information regarding PVL anatomy and location.

\section{Paravalvular leakage severity}

Considering that the major determinant of surgery is severity of PVL, the size of PVL on CT can be useful information in deciding the necessity of reoperation. Moreover, the measured size of PVL on CT was significantly correlated with the severity estimated on TTE and TEE. (Spearman's Correlation Coefficient, $r$ $=0.83, \mathrm{P}$ value $=0.0014)$.

In line with our study, Suh et al. reported that PVL size across mitral prosthesis measured on CT and PVL severity on TTE and TEE showed a positive correlation $(P<0.05)[17]$.

\section{Prosthetic valve endocarditis and periannular findimgs}

TEE had better diagnostic accuracy as compared to CT in detection of vegetations. The limited temporal resolution of the $\mathrm{CT}$ and small sized vegetations were considered common reasons for missing vegetations by $\mathrm{CT}$.

In consistent with our findings, Fagman et al and Habets et al concluded the complementary role of CT to TEE for diagnosis of vegetation $[19,20]$.

Both CT and TEE diagnosed presence of aortic root abscess in seven patients with sensitivity $100 \%$ and specificity $100 \%$ for both modalities (Intraoperative findings were considered the gold standard). These results were closely correlated to Feuchtner et al results which revealed that the diagnostic performance of CT for the detection of abscesses/pseudoaneurysms combined was sensitivity $100 \%$, specificity $100 \%$ by CT and sensitivity $89 \%$, specificity $100 \%$ by TEE [15].

\section{Occluder Function}

Both imaging modalities had excellent agreement for diagnosing occluder malfunction with sensitivity and specificity $100 \%$.

In our study, CT overcame the limitation of acoustic shadowing of mechanical prosthesis and helped identification of thrombus on mechanical prosthesis in mitral position as cause of prothesis malfunction.

These findings were strongly correlated with findings of Konen et al and LaBounty et al (Evaluation of mechanical prothesis and opening angle with MDCT [21,22].

In consistent with or findings, Feuchtner et al reported role of CTA for identification of cause of prosthesis malfunction using surgery as the reference standard [23]. 


\section{Limitations Of The Study}

The study had a relatively small sample size. We excluded patients with ball and cage prothesis due to heavy beam artefacts interfering with CT image interpretation. $3 \mathrm{D}$ echo was not performed for all enrolled patients, however there are specific limitations for 3D echo as need of optimal image quality (limited in postoperative patients), several artifacts (dropout and stitches) that may be misinterpreted as valvular perforations and periprosthetic leaks in 3 D echo [24 -27]. In our study, we exclusively enrolled patients with mechanical valves because of limited availability of bio-prosthetic valves.

\section{Conclusion}

This study demonstrates that cardiac CT showed comparable diagnostic accuracy to TEE and intraoperative findings for the detection, localization, and assessment of severity of prosthetic PVL. Moreover, CT was shown to be useful in detection of other findings related to prosthetic cardiac valves.

\section{Declarations}

\section{Funding}

"The authors declare that no funds, grants, or other support were received during the preparation of this manuscript."

\section{Competing interests}

"The authors have no relevant financial or non-financial interests to disclose."

\section{Author Contributions}

"All authors contributed to the study conception and design. Material preparation, data collection and analysis were performed by Amr Youssef Hassan, [Hussien Heshmat Kassem] and [Mohamed Ali Salem]. The first draft of the manuscript was written by [Amr Youssef Hassan]. This was revised by [Hussien Heshmat kassem] and [Hossam Ibrahim Kandil]. Statistical analysis was performed by [Amr Youssef Hassan] and [Hussien Heshmat Kassem]. All authors commented on previous versions of the manuscript. All authors read and approved the final manuscript."

\section{Compliance of ethical standard}

"This is an observational study. The Cairo university hospital Research Ethics Committee has confirmed that no ethical approval is required."

\section{Consent to participate}

"Informed consent was obtained from all individual participants included in the study." 


\section{References}

1. Hammermeister K, Sethi GK, Henderson WG, Grover FL, Oprian C, Rahimtoola SH (2000) Outcomes 15 years after valve replacement with a mechanical versus a bioprosthetic valve: final report of the Veterans Affairs randomized trial. J Am Coll Cardiol 36:1152-1158. https://. doi

2. Ionescu A, Fraser AG, Butchart EG (2003) Prevalence and clinical significance of incidental paraprosthetic valvar regurgitation: a prospective study using transoesophageal echocardiography. Heart 89:1316-1321. https://doi.org/10.1136/heart.89.11.1316)

3. Cho IJ, Moon J, Shim CY, Jang Y, Chung N, Chang BC, Ha JW (2011) Different clinical outcome of paravalvular leakage after aortic or mitral valve replacement. Am J Cardiol 107:280-284. https://. doi

4. Dávila-Román VG, Waggoner AD, Kennard ED, Holubkov R, Jamieson WR, Englberger L, Carrel TP, Schaff HV (2004) Artificial Valve Endocarditis Reduction Trial echocardiography study. Prevalence and severity of paravalvular regurgitation in the Artificial Valve Endocarditis Reduction Trial (AVERT) echocardiography study. J Am Coll Cardiol 44:1467-1472. https://. doi

5. Rallidis LS, Moyssakis IE, Ikonomidis I, Nihoyannopoulos P (1999) Natural history of early aortic paraprosthetic regurgitation: a five-year follow-up. Am Heart J 138(2 pt 1):351-357. https://doi.org/10.1016/S0002-8703(99)70124-9)

6. Flachskampf FA, Hoffmann R, Franke A, Job FP, Schöndube FA, Messmer BJ, Hanrath P (1995) Does multiplane transesophageal echocardiography improve the assessment of prosthetic valve regurgitation? J Am Soc Echocardiogr 8:70-78. https://doi.org/10.1016/S0894-7317(05)80360-8)

7. Daniel LB, Grigg LE, Weisel RD, Rakowski H (1990) Comparison of transthoracic and transesophageal assessment of prosthetic valve dysfunction. Echocardiography 7:83-95. https://doi.org/10.1111/j.1540-8175.1990.tb00352.x)

8. Hassan AY, Kassem HH, Kandil HI, Meshaal MS, Salem MA, Mohamed AS (2021) Impact of Cardiac Computed Tomographic Angiography on Diagnostic and Therapeutic Decisions in Patients with Suspected Prosthetic Heart Valve Dysfunction. Journal of the Saudi Heart Association 33(1):85. https://. doi

9. Kliger C, Eiros R, Isasti G, Einhorn B, Jelnin V, Cohen H, Kronzon I, Perk G, Fontana GP, Ruiz CE (2013) Review of surgical prosthetic paravalvular leaks: diagnosis and catheter-based closure. Eur Heart $\mathrm{J}$ 34:638-649. https://. doi

10. O’Neill AC, Martos R, Murtagh G, Ryan ER, McCreery C, Keane D, Quinn M, Dodd JD (2014) Practical tips and tricks for assessing prosthetic valves and detecting paravalvular regurgitation using cardiac CT. J Cardiovasc Comput Tomogr 8:323-327. https://. doi

11. Habets J, Meijer TS, Meijer RC, Mali WP, Vonken EJ, Budde RP (2012) CT attenuation measurements are valuable to discriminate pledgets used in prosthetic heart valve implantation from paravalvular leakage. Br J Radiol 85:e616-e621. https:// doi: 10.1259/bjr/29602784) 
12. Ruiz CE, Jelnin V, Kronzon I, Dudiy Y, Del Valle-Fernandez R, Einhorn BN, Chiam PT, Martinez C, Eiros R, Roubin G, Cohen HA (2011) Clinical outcomes in patients undergoing percutaneous closure of periprosthetic paravalvular leaks. J Am Coll Cardiol 58:2210-2217. https://. doi

13. Lang RM, Bierig M, Devereux RB et al (2005) Recommendations for chamber quantification: a report from the American Society of Echocardiography's Guidelines and Standards Committee and the Chamber Quantification Writing Group, developed in conjunction with the European Association of Echocardiography, a branch of the European Society of Cardiology. Journal of the American Society of Echocardiography. Dec 1;18(12):1440-63. (https://doi.org/10.1016/j.echo.2005.10.005)

14. Zoghbi WA, Chambers JB, Dumesnil JG et al (2009) Recommendations for evaluation of prosthetic valves with echocardiography and Doppler ultrasound: a report from the American Society of Echocardiography's Guidelines and Standards Committee and the Task Force on Prosthetic Valves, developed in conjunction with the American College of Cardiology Cardiovascular Imaging Committee, Cardiac Imaging Committee of the American Heart Association, the European Association of Echocardiography, a registered branch of the European Society of Cardiology, the Japanese.Journal of the American Society of Echocardiography. Sep 1;22(9):975-1014.

(https://doi.org/10.1016/j.echo.2009.07.013)

15. Feuchtner GM, Stolzmann P, Dichtl W et al Multislice computed tomography in infective endocarditis: comparison with transesophageal echocardiography and intraoperative findings. Journal of the American College of Cardiology. 2009 Feb 3;53(5):436-44.

(https://doi.org/10.1016/j.jacc.2008.01.077)

16. Hara $\mathrm{M}$, Nishino $\mathrm{M}$, Taniike $\mathrm{M}$, Makino $\mathrm{N}$ et al Impact of 64 multi-detector computed tomography for the evaluation of aortic paraprosthetic regurgitation. Journal of cardiology. 2011 Nov 1;58(3):294-9. (https://doi.org/10.1016/j.jjcc.2011.08.002)

17. Suh YJ, Hong GR, Han K et al (2016 Jun) Assessment of mitral paravalvular leakage after mitral valve replacement using cardiac computed tomography: comparison with surgical findings. Circulation: Cardiovascular Imaging 9(6):e004153.

https://doi.org/10.1161/CIRCIMAGING.115.004153)

18. Montorsi P, Cavoretto D, Repossini A et al (1996 Jan) Valve design characteristics and cinefluoroscopic appearance of five currently available bileaflet prosthetic heart valves. Am J Card Imaging 10(1):29-41

19. Fagman E, Perrotta S, Bech-Hanssen $O$ et al (2012) ECG-gated computed tomography: a new role for patients with suspected aortic prosthetic valve endocarditis. European radiology. Nov 1;22(11):240714. (. 10.1007/s00330-012-2491-5)

20. Habets J, Tanis W, van Herwerden LA et al Cardiac computed tomography angiography results in diagnostic and therapeutic change in prosthetic heart valve endocarditis. The international journal of cardiovascular imaging. 2014 Feb 1;30(2):377-87. (https://doi 10.1007/s10554-013-0335-2)

21. Konen E, Goitein O, Feinberg MS et al The role of ECG-gated MDCT in the evaluation of aortic and mitral mechanical valves: initial experience.American Journal of Roentgenology. 2008Jul; 
191(1):26-31. ( https://doi:10.2214/AJR.07.2951)

22. LaBounty TM, Agarwal PP, Chughtai A et al (2009 Nov) Evaluation of mechanical heart valve size and function with ECG-gated 64-MDCT. American Journal of Roentgenology 193(5):W389-W396. https://doi:10.2214/AJR.08.2178)

23. Feuchtner G, Plank F, Mueller S et al Cardiac CTA for evaluation of prosthetic valve dysfunction. JACC: Cardiovascular Imaging. 2017 Jan 2;10(1):91-3. (http://dx.doi.org/10.1016/j.jcmg.2016.08.008)

24. Le HT, Hangiandreou N, Timmerman R et al (2016) Imaging artifacts in echocardiography. Anesthesia \& Analgesia. Mar 1;122(3):633-46. (https://doi.org/10.1213/ANE.0000000000001085)

25. Faletra FF, Ramamurthi A, Dequarti MC et al (2014) Artifacts in three-dimensional transesophageal echocardiography. Journal of the American Society of Echocardiography. May 1;27(5):453-62. (https://doi.org/10.1016/j.echo.2014.02.003)

26. Mahmood F, Jeganathan J, Saraf $R$ et al A practical approach to an intraoperative three-dimensional transesophageal echocardiography examination. Journal of cardiothoracic and vascular anesthesia. 2016 Apr 1;30(2):470-90. (https://doi.org/10.1053/j.jvca.2015.10.014)

27. Saxena P, Malouf JF, Click R et al (2014 Jan) 3 D Echocardiography in Cardiac Surgery. Journal of Cardiac Surgery: Including Mechanical and Biological Support for the Heart and Lungs. 29:51-54. https://doi.org/10.1111/jocs.12256. 1

\section{Tables}

Table 1. Population characteristics 


\begin{tabular}{|c|c|}
\hline variable & No. (\%) \\
\hline Mean Age (years) & $41 \pm 12$ \\
\hline \multicolumn{2}{|l|}{ Gender } \\
\hline Male & $19(73 \%)$ \\
\hline Female & $7(27 \%)$ \\
\hline \multicolumn{2}{|l|}{ Prosthetic site } \\
\hline Mitral & $10(38 \%)$ \\
\hline Aortic & $8(31 \%)$ \\
\hline Both & $8(31 \%)$ \\
\hline \multicolumn{2}{|l|}{ Prosthetic Type } \\
\hline Mechanical & $26(100 \%)$ \\
\hline \multicolumn{2}{|l|}{ Duration from last surgery } \\
\hline Less than one year & $14(54 \%)$ \\
\hline More than one year & $12(46 \%)$ \\
\hline \multicolumn{2}{|l|}{ Blood culture: } \\
\hline Positive & $8(31 \%)$ \\
\hline Negative & $7(27 \%)$ \\
\hline Not done & $11(42 \%)$ \\
\hline \multicolumn{2}{|l|}{ Serology: } \\
\hline Positive & $10(39 \%)$ \\
\hline Negative & $5(19 \%)$ \\
\hline Not done & $11(42 \%)$ \\
\hline Mean Hemoglobin level (g/dl) & $9.0 \pm 2.09$ \\
\hline Mean Total leucocytic count $\left(10^{3} / \mathrm{mm}\right)$ & $10.6 \pm 6.02$ \\
\hline Mean creatinine (mg/dl) & $0.82 \pm 0.27$ \\
\hline Mean CRP level (mg/L) & $53.16 \pm 6.89$ \\
\hline Mean ESR level (mm/hr) & $66.5 \pm 36.0$ \\
\hline
\end{tabular}


Table 2. Agreement analysis between CT and TEE for Paravalvular leakage presence and location

\begin{tabular}{|llll|}
\hline Variables & CT (no.) & TEE (no.) & Agreement (k) \\
\hline PVL presence & 25 & 26 & 0.96 \\
\hline PVL location & & & \\
\hline Anteromedial (mitral) & 2 & 2 & 1 \\
Posteromedial (mitral) & 1 & 1 & 1 \\
Anterolateral (mitral) & 3 & 3 & 1 \\
Posterolateral (mitral) & 6 & 6 & 1 \\
Anterior occluder (aorta) & 8 & 9 & 0.96 \\
Posterior occluder (aorta) & 5 & 5 & 1 \\
\cline { 2 - 4 } & & &
\end{tabular}

Table 3. Correlation between paravalvular leakage defect size on CT and paravalvular leakage severity on echocardiography

\begin{tabular}{|lll|}
\hline Variable & Defect size by CT $(\mathrm{mm})$ Spearman's Correlation, $r$ & P Value* $^{\star}$ \\
\hline Severity by TEE & 0.83 & 0.0014 \\
\hline
\end{tabular}

* $P$ value $<0.05$ statistically significant

Table 4. Diagnostic performances of echocardiography and CT for the demonstration of prosthetic valve dysfunction as compared with surgical findings

\begin{tabular}{|lllll|}
\hline Variables & \multicolumn{5}{c|}{ Echocardiography } & CT \\
\hline Vegetation & $7(\mathrm{TP})$ & $0(\mathrm{FP})$ & $6(\mathrm{TP})$ & $0(\mathrm{FP})$ \\
\cline { 2 - 5 } & $1(\mathrm{FN})$ & $18(\mathrm{TN})$ & $2(\mathrm{FN})$ & $16(\mathrm{TN})$ \\
\hline Abscess & $7(\mathrm{TP})$ & $0(\mathrm{FP})$ & $7(\mathrm{TP})$ & $0(\mathrm{FP})$ \\
\hline pseudoaneurysm & $0(\mathrm{FN})$ & $19(\mathrm{TN})$ & $0(\mathrm{FN})$ & $19(\mathrm{TN})$ \\
\hline \multirow{2}{*}{ Occluder malfunction } & $0(\mathrm{TP})$ & $0(\mathrm{FP})$ & $1(\mathrm{TP})$ & $0(\mathrm{FP})$ \\
\cline { 2 - 5 } & $1(\mathrm{FN})$ & $25(\mathrm{TN})$ & $0(\mathrm{FN})$ & $25(\mathrm{TN})$ \\
\cline { 2 - 5 } & $0(\mathrm{FN})$ & $0(\mathrm{FP})$ & $1(\mathrm{TP})$ & $0(\mathrm{FP})$ \\
\cline { 2 - 5 } & & $25(\mathrm{TN})$ & $0(\mathrm{FN})$ & $25(\mathrm{TN})$ \\
\hline
\end{tabular}


TP: True positive, FN: False negative, FP: False positive, TN: True negative

\section{Figures}

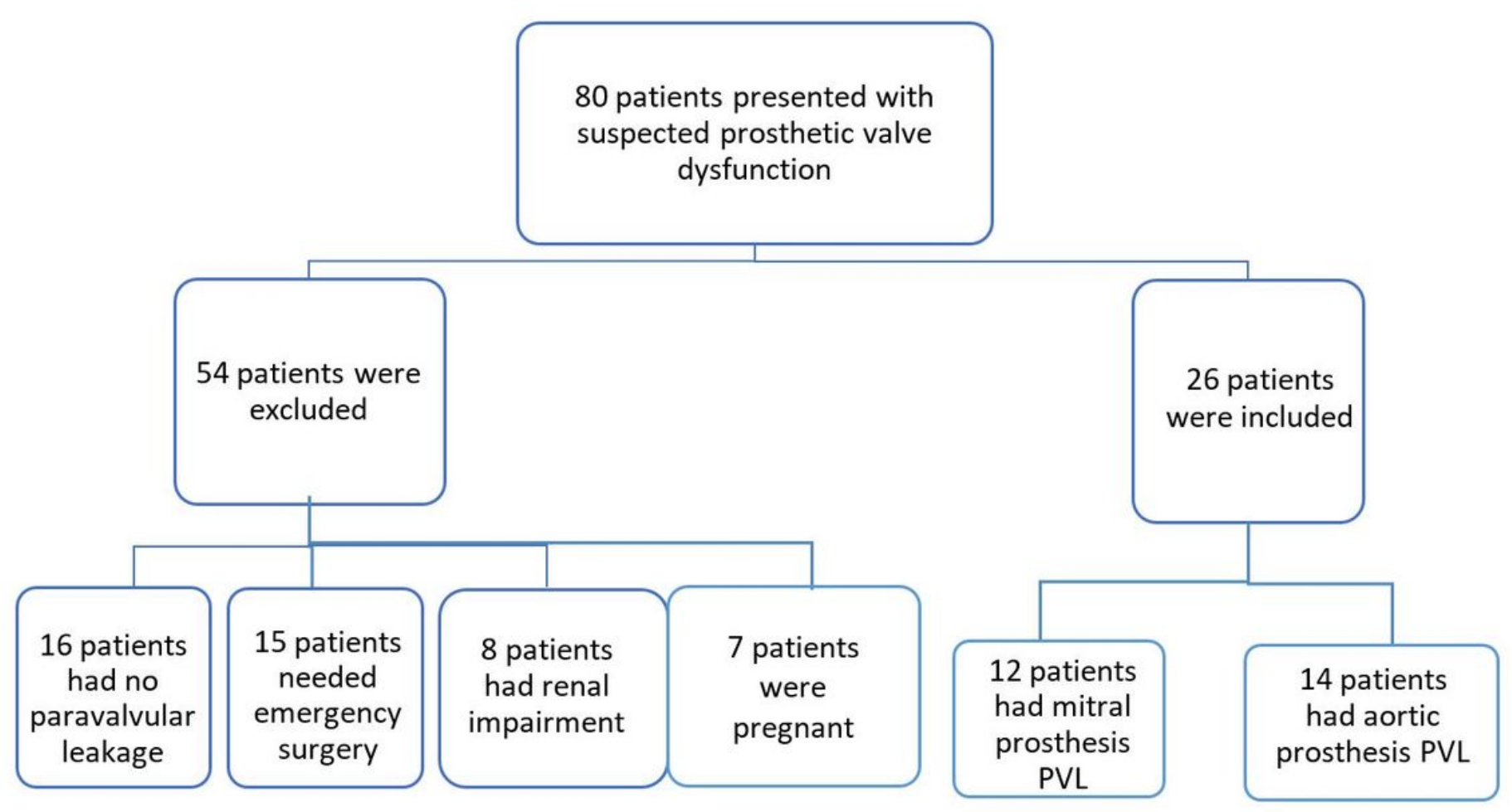

\section{Figure 1}

Diagram for enrolled patients 

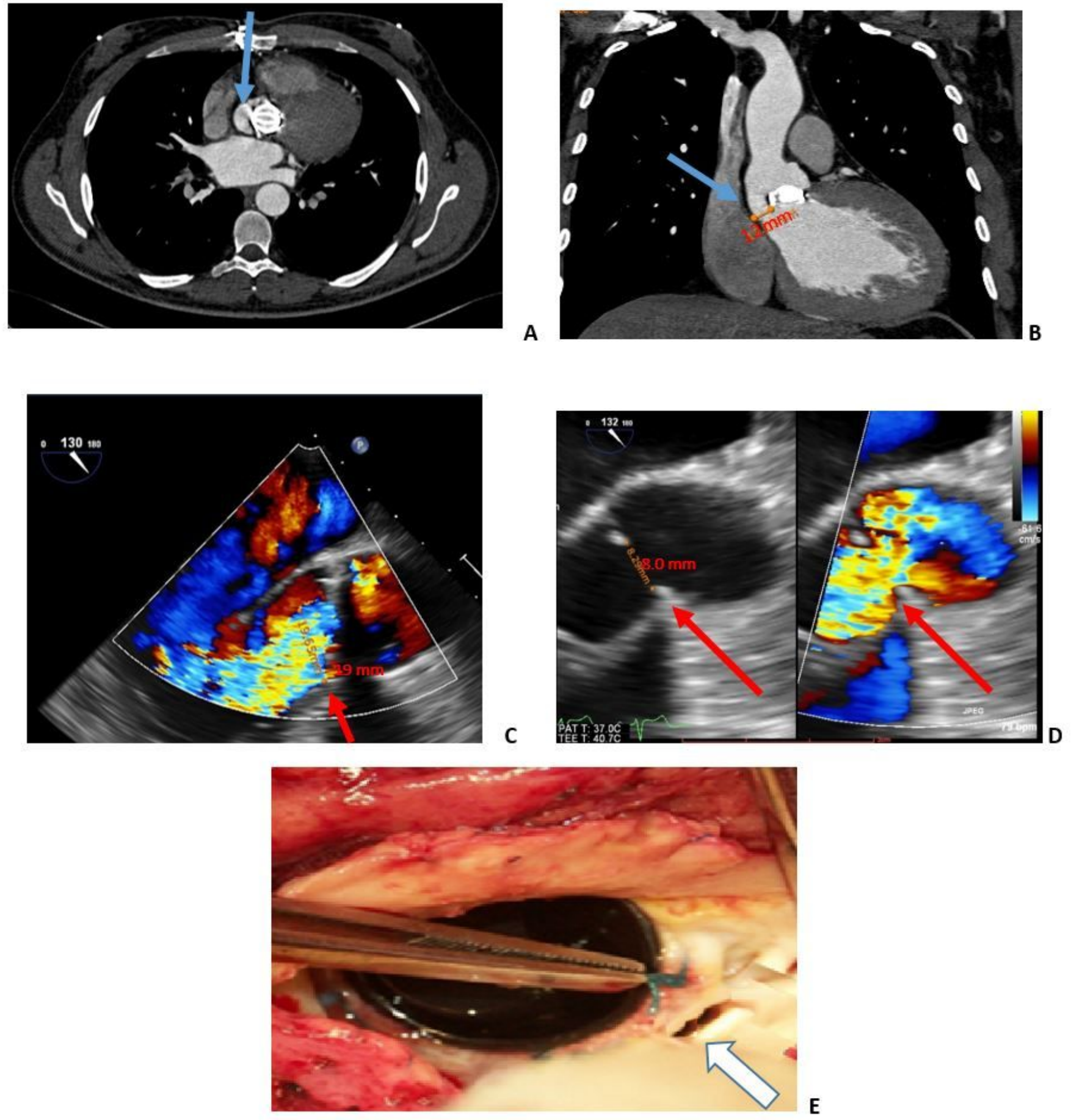

Figure 2

CT and TEE in a patient with severe aortic prosthetic paravalvular leak and heart failure symptoms $(\mathrm{A})$ : MCTA, Axial cut, short axis view showing aortic prosthetic PVL across the lateral aspect of aortic bileaflet mechanical prosthesis (blue arrow) (B): MCTA, coronal cut, showed defect perimeter measuring $12 \mathrm{~mm}$ (blue arrow) related to one of occluder of aortic prosthesis. (C, D): TEE, mid esophageal, long axis view, showing paravalvular regurgitation related to posterior aspect of aortic mechanical prosthesis with jet 
width measuring $19 \mathrm{~mm}$ and occupies almost whole LVOT and defect size $8 \mathrm{~mm}$ (red arrow) by TEE. (E): surgical image showing aortic prosthesis PVL defect for the same patient (white arrow)
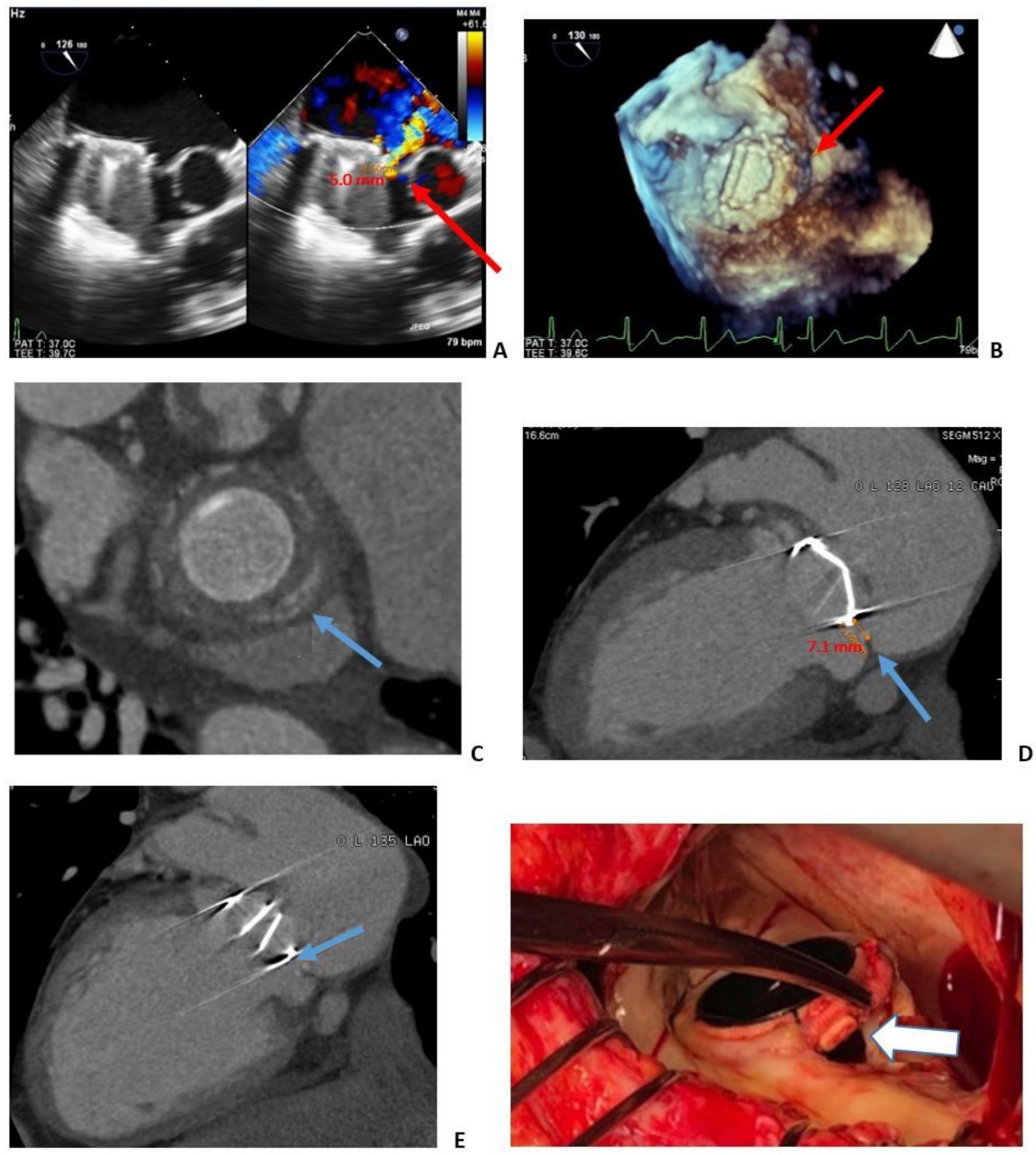

Figure 3

TEE and CT for patient with significant mitral prosthetic paravalvular leak and heart failure symptoms (A): TEE, mid esophageal, long axis view, showing moderate paravalvular regurgitation related to one of 
the occlders of mitral mechanical prosthesis with vena contracta measuring $5.0 \mathrm{~mm}$ (red arrow) (B): TEE, enface view, 3-D image showing the defect related to the mitral mechanical prosthesis (red arrow) (C): MCTA, Axial cut, short axis view showing defect of PVL related to posteromedial aspect of mitral mechanical prosthesis (blue arrow) (D): MCTA, Coronal cut, long axis view, systolic phase showing defect perimeter of mitral prosthetic PVL measuring $7.1 \mathrm{~mm}$ (blue arrow) (E): MCTA, Coronal cut, long axis view, Diastolic phase showing defect of mitral prosthetic PVL (blue arrow) (F): surgical image showing mitral prosthesis PVL defect for the same patient (white arrow). 